\title{
Mechanical Characterization of High Aspect Ratio Silicon Nanolines
}

Bin $\mathrm{Li}^{1}$, Huai Huang ${ }^{1}$, Qiu Zhao ${ }^{1}$, Zhiquan Luo ${ }^{1}$, Jang-Hi $\mathrm{Im}^{1}$, Paul S Ho ${ }^{1}$, Min Kyoo Kang ${ }^{2}$, Rui Huang $^{2}$, and Michael W Cresswell ${ }^{3}$

${ }^{1}$ Laboratory for Interconnect and Packaging, Microelectronics Research Center, University of Texas, Austin, TX, 78758

${ }^{2}$ Department of Aerospace Engineering and Engineering Mechanics, University of Texas, Austin, TX, 78712

${ }^{3}$ M\&K Single-Crystal Standards, Frederick, MD, 21702

\section{ABSTRACT}

In this study, we performed nanoindentation experiments on two sets of silicon nanolines (SiNLs) of widths $24 \mathrm{~nm}$ and $90 \mathrm{~nm}$, respectively, to investigate the mechanical behavior of silicon structures at tens of nanometer scale. The high height-to-width aspect ratio $(\sim 15)$ SiNLs were fabricated by an anisotropic wet etching (AWE) method, having straight and nearly atomically flat sidewalls. In the test, buckling instability was observed at a critical load, which was fully recoverable upon unloading. It was found that friction at the contact between the indenter and SiNLs played an important role in the buckling response. Based on a finite element model (FEM), the friction coefficient was estimated to be in a range of 0.02 to 0.05 . The strain to failure was estimated to range from $3.8 \%$ for $90 \mathrm{~nm}$ lines to $7.5 \%$ for $24 \mathrm{~nm}$ lines.

\section{INTRODUCTION}

Silicon-based nanostructures are essential building blocks for nanoelectronic devices and nano-electromechanical systems (NEMS). Fabrication and mechanical characterization of silicon nanostructures have attracted particular interest in recent years. For example, Hoffmann et al. reported an average strain to failure of $6 \%$ and a fracture strength of $12 \mathrm{GPa}$ [1] for silicon nanowires (SiNWs) with diameters between $90 \mathrm{~nm}$ and $200 \mathrm{~nm}$. The measured strength was significantly higher than those for microscale Si beams (4 GPa) [2] and millimeter scale Si beams ( $\sim 500 \mathrm{MPa})$ [3]. Due to delicate requirements on sample handling, transducer resolution, and the interpretation of measurement data, characterization of mechanical properties at the tens of nanometer scale is still of great challenge [4,5].

Owing to the large surface to volume ratio, friction at contact is of fundamental importance for reliability of micro/nano-devices, such as NEMS and hard-disk drives [6,7]. It has been reported that there was a transition of frictional shear strength, decreasing by almost one order of magnitude as the contact radii shrank into the nanoscale range [8]. This indicates a size effect at contact on friction properties.

In this paper, we extended the study of mechanical properties of nanostructures to the tens of nanometer range, by conducting nanoindentation tests on two sets of high aspect ratio $(\sim 15)$ SiNLs. An AWE process was first used to fabricate the SiNLs, having smooth sidewalls and well-defined cross sections. Buckling instability was investigated in the indentation tests, and FEM simulation was used for the extraction of material properties of the SiNLs, e.g., friction coefficient, strain to failure, etc. It was found that the buckling behavior of these SiNLs was 
dependent on the combined effects of load, line geometry, mechanical properties of SiNLs, and the friction properties at contact.

\section{EXPERIMENTAL APPROACH}

The SiNLs were fabricated on a (110) SOI wafer using a process combining electronbeam lithography (EBL) and anisotropic wet etching (AWE) [9]. AWE is a pattern transfer method based on the large etching rate differences among different crystalline planes [10]. When combined with high-resolution EBL, it can yield very high-quality SiNLs on a (110) wafer. Figure 1 shows the scanning electron microscope (SEM) images of the parallel SiNLs with 24 $\mathrm{nm}$ and $90 \mathrm{~nm}$ line widths. The line dimensions are summarized in Table 1. Although the aspect ratio (AR) was higher than 15, these nanolines possessed well-defined geometry with smooth sidewalls, and the highly uniform line widths.
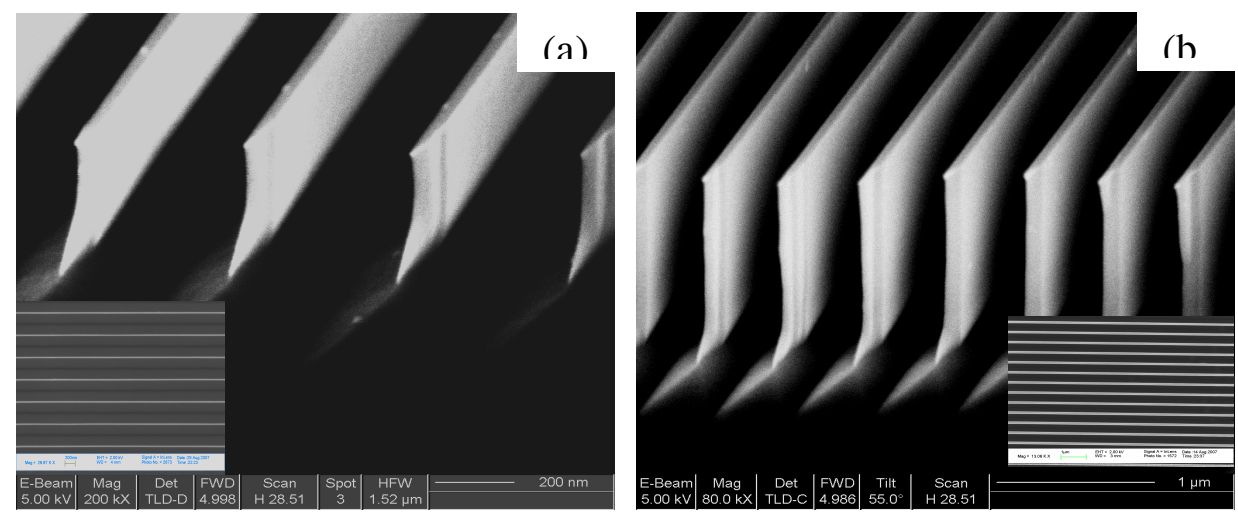

Figure 1. Cross-sectional SEM images of the two sets of high aspect ratio SiNLs. Tilt angle is $60^{\circ}$. (a) $24 \mathrm{~nm}$ wide lines (b) $90 \mathrm{~nm}$ wide lines. A trench pattern was specially designed at one end of the lines to facilitate the cross-sectional SEM imaging, showing the sharp edges due to AWE. Insets are plan-view images of the SiNLs.

Table 1 Dimensions of the two sets of SiNLs fabricated for indentation tests.

\begin{tabular}{|c|c|c|c|c|c|}
\hline Line Dimension (nm) & Width & Height & Pitch & Trench Width & Aspect ratio \\
\hline SiNL set 1 & 24 & 380 & 390 & 366 & 15.8 \\
\hline SiNL set 2 & 90 & 1400 & 450 & 360 & 15.6 \\
\hline
\end{tabular}

An AFM based nanoindentation system (Triboscope by Hysitron, Inc.) was used to characterize the mechanical responses of the SiNLs. A conically shaped indenter with the tip radius around $3.5 \mu \mathrm{m}$ (as determined from SEM imaging) was used to probe the parallel SiNLs. Prior to indentation, the indenter was placed directly above the center area $( \pm 5 \mu \mathrm{m})$ of a $30 \mu \mathrm{m}$ by $30 \mu \mathrm{m}$ array of parallel nanolines. A contact mode AFM scan on the SiNLs was performed with the indenter tip to locate the center of a line trench as the position for the subsequent indentation test. A trapezoidally shaped loading profile was then used as the control of the indentation tests, with a dwelling time of 1s at the peak load. Load-controlled indentation tests were performed and the force vs. displacement curves were recorded for both loading and unloading processes. 


\section{EXPERIMENTAL RESULTS}

Figure 2 shows two sets of force-displacement curves obtained from nanoindentation on the $24 \mathrm{~nm}$ lines. Similar to the previous experiments on the $74 \mathrm{~nm}$ SiNLs [9], a displacement burst was observed at a critical load, which was attributed to buckling of the high aspect ratio SiNLs. In these tests the displacement was fully recovered with no residual deformation after withdrawal of the indenter. Interestingly, we observed two different deformation modes.
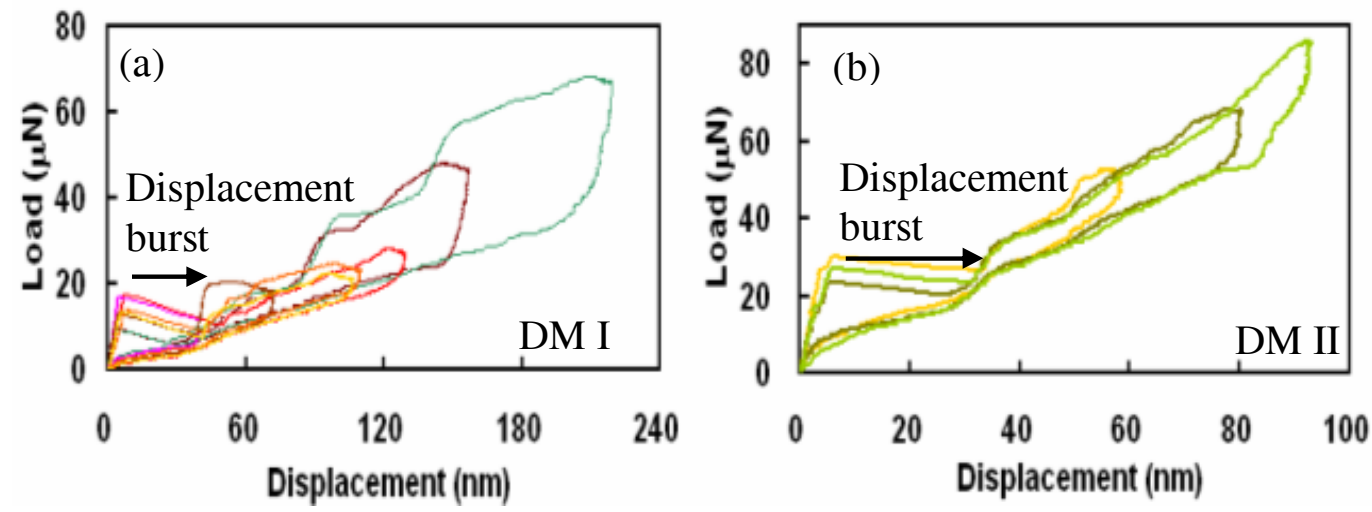

Figure 2. Load vs. displacement curves of nanoindentation tests of the $24 \mathrm{~nm}$ SiNLs. (a) DM I: critical load ranged from $9 \mu \mathrm{N}$ to $17 \mu \mathrm{N}$. (b) DM II: critical load ranged from $24 \mu \mathrm{N}$ to $30 \mu \mathrm{N}$.
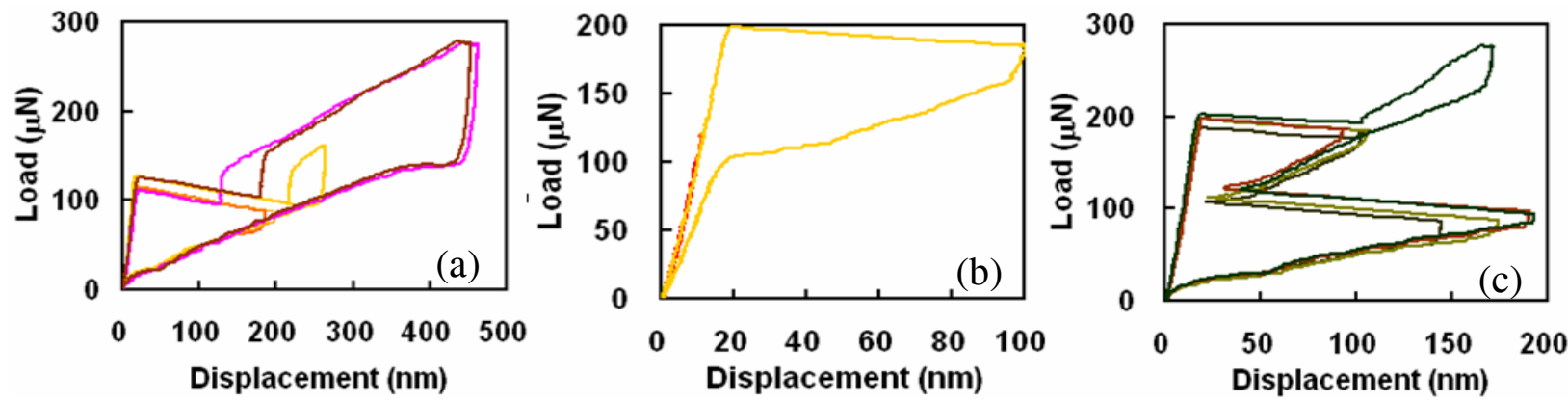

Figure 3. Load vs. displacement curves of nanoindentation tests of the $90 \mathrm{~nm}$ SiNLs. (a) DM I: Critical load was $\sim 120 \mu \mathrm{N}$. (b) DM II.1: critical load $\sim 200 \mu \mathrm{N}$. (c) DM II.2: critical load $\sim 200$ $\mu \mathrm{N}$ with the curves having a second displacement burst during the unloading process.

In Deformation Mode (DM) I shown in Figure 2(a), the critical load ranged from $9 \mu \mathrm{N}$ to $17 \mu \mathrm{N}$. The displacement of the indenter increased to $\sim 220 \mathrm{~nm}$ under a $\sim 70 \mu \mathrm{N}$ force. This maximum indentation displacement was large, about 58\% of the line height. In DM II (Figure 2(b)), the critical loads jumped into a range of $24 \mu \mathrm{N}$ to $30 \mu \mathrm{N}$. And under the same $70 \mu \mathrm{N}$ force the displacement was only around $80 \mathrm{~nm}$, showing a much higher stiffness compared with DM I. Since the indentation tests were performed at different locations around the center area of the SiNL array, these different deformation modes were attributed to the variation of local surface conditions, in particular, the friction properties at each indentation contact.

Figure 3 shows force-displacement curves obtained from nanoindentation tests on the 90 $\mathrm{nm}$ wide SiNLs. Again, the displacement was fully recovered with no residual deformation after 
withdrawal of the indenter. Based on the critical loads, the deformation behavior can also be classified into two major modes. In DM I as shown in Figure 3(a), the critical load was around $117 \mu \mathrm{N}$. The maximum indentation displacement was $\sim 450 \mathrm{~nm}$, which was about $1 / 3$ of the line height. For DM II in Figure 3(b) and (c), the critical load was $\sim 200 \mu N$, and the maximum indentation displacement was below $200 \mathrm{~nm}$. Interestingly, it was observed that in some indentation curves of DM II, a second displacement burst occurred during the unloading process as shown in Figure 3(c). These different indentation behaviors will be further discussed with a finite element analysis (FEA) model.

\section{DISCUSSIONS}

In the FEA model [9], the indenter and the silicon substrate underneath the SiNLs were both assumed to be rigid. The indenter was located on the pattern trench center, simulating the experimental setup. In simulations, a vertical displacement was applied to the reference node of the indenter and the indentation force was obtained as the reaction force at the reference node. The friction at the contact of the indenter and the SiNLs was simulated by a simple Coulomb friction model, with the friction coefficient varying from 0 , i.e. frictionless, to 0.10 to investigate the influence of friction on the indentation responses.

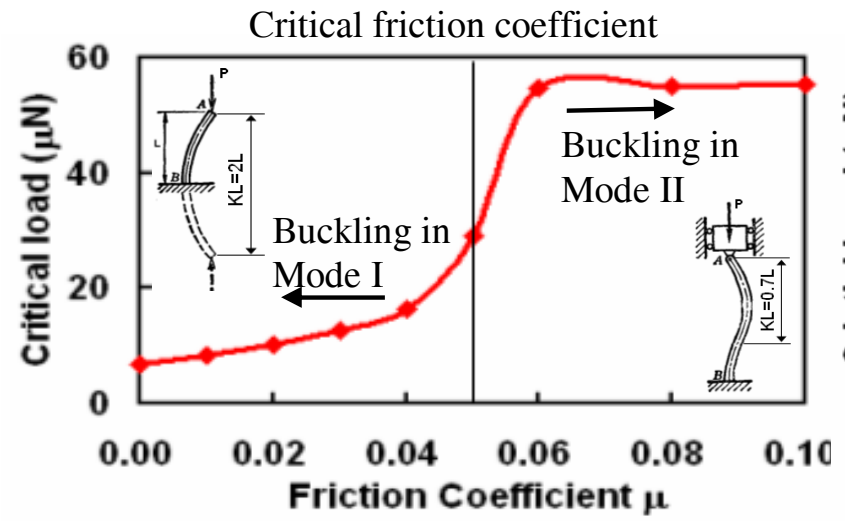

(a)

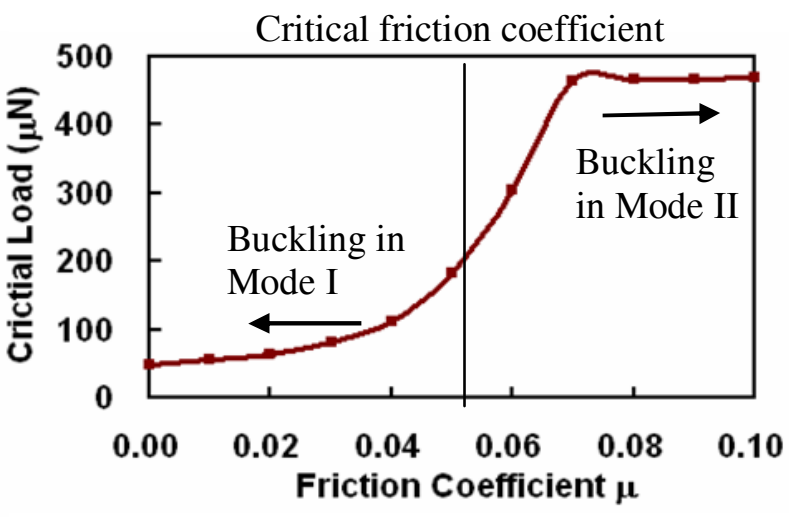

(b)

Figure 4. The critical indentation load predicted by FEA models, as a function of the friction coefficient between the indenter tip and SiNLs. (a) $24 \mathrm{~nm}$ SiNLs. (b) $90 \mathrm{~nm}$ SiNLs.

Figure 4(a) plots the calculated critical load of indentation on $24 \mathrm{~nm}$ SiNLs as a function of the friction coefficient $\mu$. It is noted that as $\mu$ increased from 0.04 to 0.05 , the calculated critical load increased significantly from $16 \mu \mathrm{N}$ to $29 \mu \mathrm{N}$. This is found to be due to a change of the buckling mode of SiNLs. Because the movement of the top ends of the SiNLs was confined by the friction force, the buckling mode of SiNLs changed from outward bending (Mode I) at low friction to inward bending (Mode II) at high friction, with the critical friction coefficient depending on the contact angle between the indenter surface and the SiNLs. Based on the critical loads measured from the indentation tests, for DM I in Figure 2(a), the $\mu$ was estimated to be around 0.02-0.04, and the SiNLs buckled in Mode I. For DM II in Figure 2(b), $\mu$ was $\sim 0.05$, which is right in the transition zone between the two buckling modes.

Similar to the above critical load analysis for $24 \mathrm{~nm}$ lines, we also estimated the friction coefficient $\mu$ for the $90 \mathrm{~nm}$ lines. According to Figure 3 and Figure 4(b), $\mu$ ranged from 0.02 to 
0.05, which also corresponded to two different buckling modes. The values are consistent with previous works $[8,9]$, showing a reduced friction strength at nano-scale.

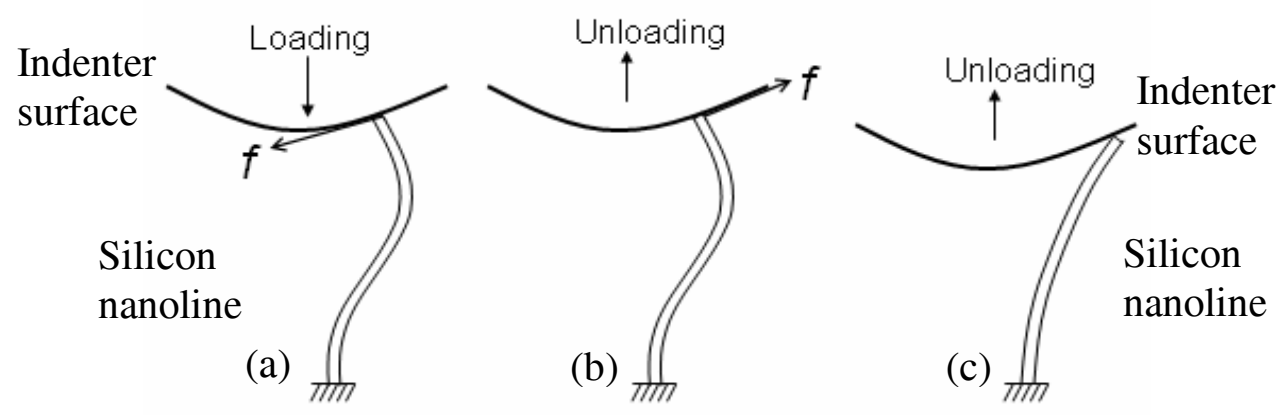

Figure 5. Schematic of the effect of friction on buckling mode transition during loading and unloading. $f$ is the friction force. Mode transfer occurred from (b) to (c) in the unloading.

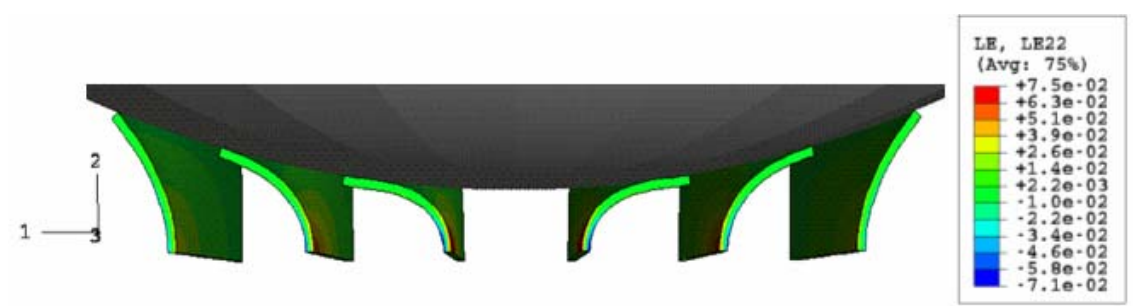

Figure 6. FEM simulation shows the deformation of the nanolines with $220 \mathrm{~nm}$ indentation displacement indentation on SiNLs.

In the indentation curves of Figure 3(c), a second displacement burst was observed during the unloading process. This behavior suggested an occurrence of a buckling mode transfer during unloading from Mode II (bending inwards) to Mode I (bending outwards). The initiation of this mode transition was attributed to the influence of friction in indentation as illustrated schematically in Figure 5. In loading, the friction force $f$ tends to suppress the transition to Mode I (Figure 5(a)). However, in the unloading process (Figure 5(b)), the friction force may change the direction and favors the transition of the buckling mode. In a load-controlled experiment, this transition leads to a displacement burst in unloading, also indicating a softening of the nanoline structure under compression (Figure 5(c)). Apparently, unlike indentation test of bulk or thin film materials where friction at the contact has a negligible influence [11], the friction property plays an important role in the indentation tests of SiNLs. It is noted that for indentation of the 24 $\mathrm{nm}$ SiNLs, in a similar DM II, the mode transfer in unloading did not occur. This could be due to a smaller recovery force for narrower lines, which is insufficient to bounce the nanoline back to its original shape to cause the mode transfer.

The FEA simulation was conducted up to the maximum indentation displacement without residual deformation in the experiments, as a lower-bound estimate of the critical strain to fracture. For $24 \mathrm{~nm}$ lines, with a $220 \mathrm{~nm}$ indentation depth the deformation of SiNLs is shown in Figure 6. The maximum principal strain of $7.5 \%$ was obtained at the bottom ends of the two center lines. Since the SiNLs did not fracture up to a $220 \mathrm{~nm}$ indentation depth, the strain to fracture of the $24 \mathrm{~nm}$ wide SiNLs was estimated to be above 7.5\%. Fracture of the SiNLs was observed in indentation tests with larger displacements, which resulted in a residual displacement 
upon unloading. With the same calculation process, the strain of failure for the $90 \mathrm{~nm}$ lines was around $3.8 \%$. These values are comparable to the published results [1,9], and also indicate a scaling effect on the fracture strength. However, more experimental data and analysis are needed to further understand the size effect.

\section{CONCLUSIONS}

In summary, nanoindentation tests together with FEA simulations were used to characterize mechanical properties of two sets of SiNLs, with height-to-width aspect ratios larger than 15 and line widths of $24 \mathrm{~nm}$ and $90 \mathrm{~nm}$. Buckling instability of the SiNLs in the indentation tests was observed and analyzed. Mechanical properties of the SiNLs were estimated from the indentation results. In particular, the friction coefficient at the contact was found to range from 0.02 to 0.05 , with such a variation possibly influenced by contact area variations and surface conditions at the nanoscale. Meanwhile, the friction property at the contact was found to play an important role in controlling the buckling behavior of SiNLs. The strain to fracture of the SiNLs was estimated ranging from $3.8 \%$ for $90 \mathrm{~nm}$ lines to $7.5 \%$ for $24 \mathrm{~nm}$ lines, both much higher than bulk Si $(\sim 1 \%)$, showing a scaling effect of the fracture strength of Si. More experimental data are needed for a statistical analysis to further understand the scale dependence of the fracture strength.

\section{ACKNOWLEDGMENTS}

The work was performed in part at the Microelectronics Research Center of the University of Texas at Austin, a member of the National Nanofabrication Infrastructure Network supported by the National Science Foundation under award No. 0335765. MKK and RH are grateful for the financial support by the National Science Foundation through Grant No. CMMI-0654105. We are also grateful to Richard Allen of the Semiconductor Electronics Division at NIST for helpful technical discussions.

\section{REFERENCES}

1. S. Hoffmann, I. Utke, B. Moser, J. Michler, S. H. Christiansen, V. Schmidt, S. Senz, P. Werner, U. Gösele, and C. Ballif, Nano Lett., 6, 622 (2006).

2. S. Johansson, J.-A. Schweitz, L. Tenerz, and J. Tiren, J. Appl. Phys., 63, 4799 (1988).

3. T. Namazu, Y. Isono, and T. J. Tanaka, J. Microelectromechanical Systems, 9, 450 (2000).

4. E. P. S. Tan, and C. T. Lim, Composites Science and Technology, 66, 1102 (2006).

5. M. Tabib-Azar, M. Nassirou, R. Wang, S. Sharma, T. I. Kamins, M. S. Islam, and R. S. Williams, Appl. Phys. Lett., 87, 113102 (2005).

6. B. Bhushan, Phil. Trans. R. Soc. A, 366, 1499 (2008).

7. E. Riedo, and H. Brune, Appl. Phys. Lett., 83, 1986 (2003).

8. D. Xu, K. Ravi-Chandar, and K. M. Liechti, J. Colloid Interface Sci., 318, 507 (2008).

9. B. Li, M. K. Kang, K. Lu, R. Huang, P. S. Ho, R. A. Allen, and M. W. Cresswell, Nano Lett., 8, 92 (2008).

10. M. W. Cresswell, W. F. Guthrie, R. G. Dixson, C. E. Murabito, and J. V. M. De Pinillos, J. Res. Natl. Inst. Stand. Technol., 111, 187 (2006).

11. H. Lee, J. H. Lee, and G. M. Pharr, J. Mech. Phys. Solid., 53, 2037 (2005). 